\title{
Lixiviação de nitrato em pastagem de coastcross adubada com nitrogênio ${ }^{1}$
}

\author{
Odo Primavesi ${ }^{2}, 4$, Ana Cândida Primavesi ${ }^{2}$, Luciano de Almeida Corrêa², Aliomar Gabriel da \\ Silva ${ }^{2}$, Heitor Cantarella ${ }^{3}$
}

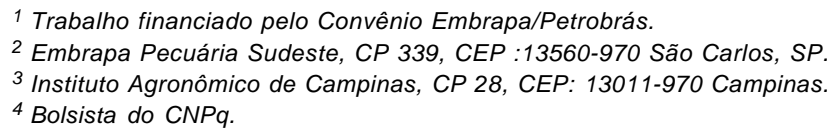

RESUMO - Objetivou-se verificar possíveis perdas de N-nitrato em profundidade, em experimentos conduzidos no período das chuvas em dois anos consecutivos em pastagem de capim-coastcross (Cynodon dactylon cv. coastcross) instalada sobre Latossolo Vermelho Distrófico típico, na região de São Carlos, SP. Foram determinados os teores de nitrato no perfil do solo, após aplicações superficiais de 125, 250, 500 e $1.000 \mathrm{~kg} \mathrm{ha}^{-1} \mathrm{ano}^{-1}$ de nitrogênio, na forma de uréia e nitrato de amônio, parcelados em cinco vezes, até a profundidade de $160 \mathrm{~cm}$. Em 1999, a determinação dos teores de N-nitrato após o quinto corte do capim, indicou maiores teores para nitrato de amônio, em especial na maior dose de N. As maiores variações ocorreram até $100 \mathrm{~cm}$. Em 2000, foram medidos semanalmente os teores de N-nitrato no perfil do solo nos tratamentos com as doses de 500 e $1.000 \mathrm{~kg} \mathrm{ha}^{-1} \mathrm{ano}^{-1} \mathrm{de} \mathrm{N}$. As maiores variações (de 0 a $81,2 \mathrm{mg} \mathrm{kg}^{-1} \mathrm{de} \mathrm{N}-\mathrm{NO}_{3}{ }^{-}$) ocorreram até $40 \mathrm{~cm}$ de profundidade. Os resultados indicam não haver risco de contaminação do lençol freático em solos profundos de textura média ocupados por gramínea tropical manejada intensivamente quando não se utilizam adubações nitrogenadas superiores à capacidade de ciclagem da forrageira e quando se considera o potencial de fornecimento do solo.

Palavras-chave: aplicação superficial, Cynodon dactylon cv. coastcross, lixiviação de nitrato, nitrato de amônio, uréia

\section{Nitrate leaching in heavily nitrogen fertilized coastcross pasture}

\begin{abstract}
The goal of this work was to assess nitrate losses in the soil profile. Nitrate concentration was determined in the profile of a soil under coastcross pasture (Cynodon dactylon cv. Coastcross), in São Carlos, SP, Brazil, in the rainy season of two consecutive years. The soil was a dark red Latosol (Hapludox), affected by tropical altitude climate. Nitrogen doses of 125, 250, 500 and 1,000 $\mathrm{kg} \mathrm{ha}^{-1} \mathrm{year}^{-1}$, as urea or ammonium nitrate were broadcasted splited in five applications. Soil nitrate concentration was determined down to $160 \mathrm{~cm}$ depth. In 1999, nitrate level varied according to $\mathrm{N}$ sources, reaching higher figure in soil that had received $1,000 \mathrm{~kg} \mathrm{ha}^{-1}$ year ${ }^{-1}$ of $\mathrm{N}$. The highest $\mathrm{NO}_{3}-\mathrm{N}$ concentration occurred in soil layer down to $100 \mathrm{~cm}$ depth, in the plots treated with ammonium nitrate. In 2000, nitrate-N was measured weekly in the soil profile, in treatments with 500 and $1,000 \mathrm{~kg} \mathrm{ha}^{-1}$ year ${ }^{-1}$. The greatest variations, from 0 to $81.2 \mathrm{mg} \mathrm{kg}^{-1} \mathrm{of} \mathrm{NO}_{3}-\mathrm{N}$, occurred down to $40 \mathrm{~cm}$ depth. Results point to low danger of water table contamination in deep medium textured soils of intensively well managed tropical grass pasture, using $\mathrm{N}$ doses lower than the forage $\mathrm{N}$ cycling capacity and considering the potential soil $\mathrm{N}$ sources.
\end{abstract}

Key Words: ammonium nitrate, application on surface, Cynodon dactylon cv. Coastcross, nitrate leaching, urea

\section{Introdução}

$\mathrm{O} \mathrm{NO}_{3}^{-}$no solo resulta diretamente do fertilizante nitrogenado aplicado ou da mineralização da matéria orgânica. Por outro lado, quando o $\mathrm{NO}_{3}{ }^{-}$na solução do solo não é absorvido pelas plantas ou imobilizado pela microbiota do solo, pode ser facilmente lixiviado, pois apresenta carga negativa e não é adsorvido pelos colóides do solo que apresentam predominantemente cargas negativas. Em condições de redução na disponibilidade de oxigênio no solo, no período das chuvas ou com irrigação, pode ocorrer perda de $\mathrm{N}-\mathrm{NO}_{3}{ }^{-}$na forma de óxido nitroso $\left(\mathrm{N}_{2} \mathrm{O}\right)$, um gás de efeito estufa com capacidade para armazenar aproximadamente 250 vezes mais energia que o $\mathrm{CO}_{2}$ (Granli \& Bøckman, 1994).

A adubação das pastagens, principalmente a nitrogenada, é um dos fatores mais importantes na determinação do nível de produção de forragem por área. A resposta das gramíneas forrageiras tropicais a altas doses de $\mathrm{N}$ tem sido relatada por vários pesquisadores (Vicente Chandler et al., 1959; Corsi, 1986; Favoreto et al., 1995). Resposta de até $1.800 \mathrm{~kg} \mathrm{ha}^{-1} \mathrm{ano}^{-1}$ de $\mathrm{N}$ foi relatada por VicenteChandler (1959), ocorrendo, porém, os maiores incrementos 
de produção na faixa de $300 \mathrm{a} 400 \mathrm{~kg} \mathrm{ha}^{-1} \mathrm{ano}^{-1} \mathrm{deN}$ (Gomes et al., 1987; Favoretto et al., 1995).

Segundo Mello et al. (1984) e Mello (1987), surgem, porém, dúvidas sobre a possibilidade de impacto ambiental negativo com essas elevadas doses de $\mathrm{N}$, haja vista o potencial de perda por lixiviação na forma de $\mathrm{N}^{-\mathrm{NO}_{3}}{ }^{-}$e a contaminação do lençol freático, especialmente em regiões com clima úmido ou em período chuvoso, justamente quando se aplicam fertilizantes nitrogenados em condições de clima tropical. Os autores afirmam ainda que este fato pode ser agravado com uso intenso de calcário, que acelera a degradação de material orgânico e estimula a nitrificação.

A redução da perda de $\mathrm{N}$-nitrato $\left(\mathrm{N}^{-\mathrm{NO}_{3}}{ }^{-}\right)$é importante por três razões: representa perda de $\mathrm{N}$ do solo disponível à planta (Errebhi et al., 1998); se perdido na forma de $\mathrm{N}_{2} \mathrm{O}$, poderá reforçar o aquecimento global e, com isso, reduzir também a água disponível, como conseqüência da maior evapotranspiração e das chuvas mais intensas que escoam; o $\mathrm{N}^{-\mathrm{NO}_{3}}{ }^{-}$lixiviado pode entrar em rios e águas subterrâneas e iniciar processo de eutroficação em ecossistemas naturais normalmente pobres em N (Primavesi et al., 2002).

Para consumo humano, são admitidas pela Legislação brasileira concentrações de até $10 \mathrm{mg} / \mathrm{L}$ de $\mathrm{N}^{-\mathrm{NO}_{3}}{ }^{-}$na água (Agudo, 1987). Primavesi et al. (2001) verificaram, em condições tropicais, em solos com $30 \%$ de argila, que as doses de nitrogênio fertilizante que geram excedentes de $\mathrm{N}-\mathrm{NO}_{3}{ }^{-}$ no solo também podem gerar acúmulo de $\mathrm{N}^{-\mathrm{NO}_{3}}{ }^{-}$na forragem, porém, não atingindo níveis tóxicos para os animais que a ingerem.

Segundo Di \& Cameron (2002), a lixiviação de $\mathrm{NO}_{3}{ }^{-} \mathrm{em}$ sistemas intensivos de produção agrícola como, por exemplo, em pastagens destinadas a gado de leite, é um dos grandes problemas ambientais em muitos países de clima temperado, pois a contaminação das fontes de água pode, a longo prazo, comprometer a sustentabilidade da produção agrícola de uma região (Cameron et al., 1997).

Em pastagem, grande quantidade de $\mathrm{NO}_{3}{ }^{-}$lixiviado é indicativo de que a quantidade de $\mathrm{N}$ que entrou no sistema excedeu os requerimentos para o crescimento vegetal. Esse suprimento ocorre usualmente com o fertilizante e com o retorno do $\mathrm{N}$ na urina dos animais, mas também inclui o $\mathrm{N}$ mineralizado da matéria orgânica e aquele proveniente da atmosfera (Whitehead, 1995).

As perdas de $\mathrm{N}-\mathrm{NO}_{3}{ }^{-}$no perfil do solo devem, então, ser monitoradas, especialmente em situações de manejo intensivo de pastagens, com elevado uso de insumos nitrogenados e corretivos de acidez, para que sejam tomadas medidas que promovam aumento da eficiência do uso do $\mathrm{N}$ aplicado com a finalidade de possibilitar o gerenciamento de um sistema de produção economicamente sustentável, de qualidade de forragem e com mínimo impacto ambiental negativo. Assim, foram realizados dois experimentos com os objetivos de determinar e monitorar o conteúdo de $\mathrm{N}-\mathrm{NO}_{3}{ }^{-}$no perfil do solo de áreas experimentais utilizadas na avaliação da aplicação de doses crescentes de $\mathrm{N}$ para produção de forragem de gramínea tropical.

\section{Material e Métodos}

Foram realizados dois experimentos, um no período de novembro de 1998 a abril de 1999 e o segundo, de novembro de 1999 a abril de 2000, em áreas contíguas de pastagem de capim-coastcross (Cynodon dactylon cv. Coastcross) submetida a períodos de corte-descanso, estabelecida em Latossolo Vermelho Distrófico típico, com $30 \%$ de argila, em São Carlos, SP, latitude $22^{\circ} 01^{\prime}$ S, longitude $47^{\circ} 54^{\prime}$ 'W e altitude de $836 \mathrm{~m}$, sob clima tropical de altitude.

As características químicas do solo na camada de 0 a $20 \mathrm{~cm}$, nas áreas do primeiro (início em novembro de 1998) e do segundo experimento (início em novembro de 1999) foram, respectivamente: 1) $\mathrm{pH}$ em $\mathrm{CaCl}_{2}=5,6 ; \mathrm{M} . \mathrm{O} .=$ $32 \mathrm{~g} \mathrm{dm}^{-3} ;$ P-resina $=27 \mathrm{mg} \mathrm{dm}^{-3} ; \mathrm{K}=5,1 \mathrm{mmol}_{\mathrm{c}} \mathrm{dm}^{-3}$; $\mathrm{Ca}=25 \mathrm{mmol}_{\mathrm{c}} \mathrm{dm}^{-3} ; \mathrm{Mg}=14 \mathrm{mmol}_{\mathrm{c}} \mathrm{dm}^{-3} ; \mathrm{CTC}$ a pH $7=$ $65 \mathrm{mmol}_{\mathrm{c}} \mathrm{d} \mathrm{m}^{-3}$; e V = 67\%; e 2) $\mathrm{pH} \mathrm{em} \mathrm{CaCl}_{2}=6,0$; M.O. = $33 \mathrm{~g} \mathrm{dm}^{-3} ;$ P-resina $=24 \mathrm{mg} \mathrm{dm}^{3} ; \mathrm{K}=4,0 \mathrm{mmol}_{\mathrm{c}} \mathrm{dm}^{-3}$; $\mathrm{Ca}=47 \mathrm{mmol}_{\mathrm{c}} \mathrm{dm}^{-3} ; \mathrm{Mg}=30 \mathrm{mmol}_{\mathrm{c}} \mathrm{dm}^{-3} ; \mathrm{CTC}$ a pH $7=$ $100 \mathrm{mmol}_{\mathrm{c}} \mathrm{dm}^{-3} ; \mathrm{e} \mathrm{V}=80 \%$.

$\mathrm{Na}$ área do primeiro experimento, foram aplicados $100 \mathrm{~kg} \mathrm{ha}^{-1}$ de $\mathrm{P}_{2} \mathrm{O}_{5}$ na forma de superfosfato simples e $30 \mathrm{~kg} \mathrm{ha}^{-1}$ de micronutrientes FTE BR-12. O potássio foi aplicado na forma de cloreto de potássio, junto com as adubações nitrogenadas, nas quantidades totais de $380 \mathrm{~kg} \mathrm{ha}^{-1} \mathrm{de} \mathrm{K}_{2} \mathrm{O}$ nos tratamentos com até $250 \mathrm{~kg} \mathrm{ha}^{-1} \mathrm{ano}^{-1}$ de N, e de $700 \mathrm{~kg} \mathrm{ha}^{-1}$ de $\mathrm{K}_{2} \mathrm{O}$ no tratamento $1.000 \mathrm{~kg} \mathrm{ha}^{-1} \mathrm{ano}^{-1}$ de $\mathrm{N}$, com base na extração esperada (pelas plantas). As datas de aplicação de $\mathrm{N}$ e $\mathrm{K}$ foram: 5/11/1998, 10/12, 5/1, 3/2 e 2/3/1999.

$\mathrm{Na}$ área do segundo experimento, foram aplicados $50 \mathrm{~kg} \mathrm{ha}^{-1}$ de $\mathrm{P}_{2} \mathrm{O}_{5}$ como superfosfato simples e $30 \mathrm{~kg} \mathrm{ha}^{-1}$ de micronutrientes FTE BR-12. O potássio, na forma de $\mathrm{KCl}$, foi aplicado por ocasião das adubações nitrogenadas nas quantidades totais de $260 \mathrm{~kg} \mathrm{ha}^{-1}$ de $\mathrm{K}_{2} \mathrm{O}$ (tratamento testemunha) e de $520 \mathrm{~kg} \mathrm{ha}^{-1}$ de $\mathrm{K}_{2} \mathrm{O}$ (tratamentos com 500 ou $1.000 \mathrm{~kg} \mathrm{ha}^{-1} \mathrm{ano}^{-1} \mathrm{de} \mathrm{N}$ ).

Odelineamento experimental em ambos os experimentos foi o de blocos casualizados com nove tratamentos organizados em esquema fatorial $(2 \times 4)+1$ (duas fontes de N: uréia e nitrato de amônio e quatro doses de N: 125, 250, 500 e 
$1.000 \mathrm{~kg} \mathrm{ha}^{-1} \mathrm{ano}^{-1}$, e uma testemunha), com quatro repetições (Gates, 1991). As doses de nitrogênio foram divididas em cinco vezes, sendo os fertilizantes aplicados após cada corte (cinco cortes, considerando o de uniformização), durante a estação das chuvas. As parcelas experimentais apresentavam 4 x $5 \mathrm{~m}$ e área útil de $6 \mathrm{~m}^{2}$ para avaliação da produção de forragem. $\mathrm{O}$ corte da forrageira ocorreu em intervalos médios de 29 e 33 dias no primeiro e segundo experimentos, respectivamente, a $10 \mathrm{~cm}$ da superfície do solo.

No primeiro experimento, iniciado em novembro de 1998, foi realizada amostragem do solo após o quinto corte, ao final do período das chuvas, em 31/3/1999. Nas parcelas que receberam 250 e $1.000 \mathrm{~kg} \mathrm{ha}^{-1} \mathrm{de} \mathrm{N}$, das duas fontes nitrogenadas, foram retiradas dez subamostras por amostra, além da testemunha, nas camadas de 0-10, 10-20, 20-40, 40-60, 60-80, 80-100, 100-120, 120-140, 140-160, 160-180 e $180-200 \mathrm{~cm}$ de profundidade. No segundo experimento, iniciado em novembro de 1999, após a primeira aplicação dos adubos nitrogenados (11 de novembro), foram retiradas, a cada sete dias, quatro subamostras de solo de cada bloco (repetição) para compor uma amostra composta das parcelas testemunha e das que receberam 500 e $1.000 \mathrm{~kg} \mathrm{ha}^{-1}$ de $\mathrm{N}$ de ambas as fontes, nas camadas de 0-10,10-20 e de 20 em $20 \mathrm{~cm}$ até $160 \mathrm{~cm}$. A avaliação semanal foi feita para se verificar a dinâmica da variação de perdas de $\mathrm{N}_{-} \mathrm{NO}_{3}{ }^{-}$no solo. As datas de corte da forrageira do segundo experimento foram: $0=10 / 11 / 99,1=14 / 12 / 99,2=10 / 1 / 00,3=14 / 2 / 00$, $4=13 / 3 / 00$ e $5=25 / 4 / 00$.
Em ambos os anos, imediatamente após a coleta, as amostras de solo foram acondicionadas em caixa térmica contendo gelo e encaminhadas ao laboratório para a extração e determinação de nitrato, segundo método descrito por Tedesco et al. (1985). Para o cálculo dos teores de $\mathrm{N}-\mathrm{NO}_{3}$, foi considerada densidade do solo de $1 \mathrm{~kg} \mathrm{dm}^{-3}$ (Primavesi et al., 2001a; Cantarella et al., 2002).

Os dados foram submetidos à análise de variância e ao teste Tukey para comparação da média dos tratamentos.

\section{Resultados e Discussão}

No experimento iniciado em novembro de 1998, os teores de $\mathrm{N}^{-\mathrm{NO}_{3}}{ }^{-}$foram maiores $(\mathrm{P}<0,01)$ para nitrato de amônio, em especial na dose de $1.000 \mathrm{~kg} \mathrm{ha}^{-1} \mathrm{ano}^{-1} \mathrm{de} \mathrm{N}$. As maiores variações entre as duas fontes ocorreram nas camadas até $100 \mathrm{~cm}(\mathrm{P}<0,01)$, embora pudesse ser verificada tendência de lixiviação de $\mathrm{N}-\mathrm{NO}_{3}{ }^{-}$em profundidades maiores apenas para o nitrato de amônio (Tabela 1, Figura 1). Este fato não é preocupante, pois as doses mais eficientes de $\mathrm{N}$ normalmente não ultrapassam $500 \mathrm{~kg} \mathrm{ha}^{-1} \mathrm{ano}^{-1}$ (Primavesi et al., 2001a), especialmente em solos com lençol freático mais profundo ou afastado de áreas de nascentes. Geralmente, considera-se que na camada de 0 a $40 \mathrm{~cm}$ ocorre mais de $80 \%$ do sistema radicular ciclador de nutrientes e que somente o nitrogênio abaixo de $80 \mathrm{~cm}$ poderia ser considerado sujeito à lixiviação, embora tenha-se verificado atividade radicular de gramíneas forrageiras até $300 \mathrm{~cm}$ (Primavesi

Tabela 1 - Teores de $\mathrm{N}-\mathrm{NO}_{3}$ no solo após cinco cortes de coastcross adubado com diferentes doses de $\mathrm{N}$ no período de 1998 a 1999 Table 1 - Soil $\mathrm{NO}_{3}-\mathrm{N}$ level, after five cuttings of coastcross grass fertilized with different $\mathrm{N}$ doses, in 1998-1999

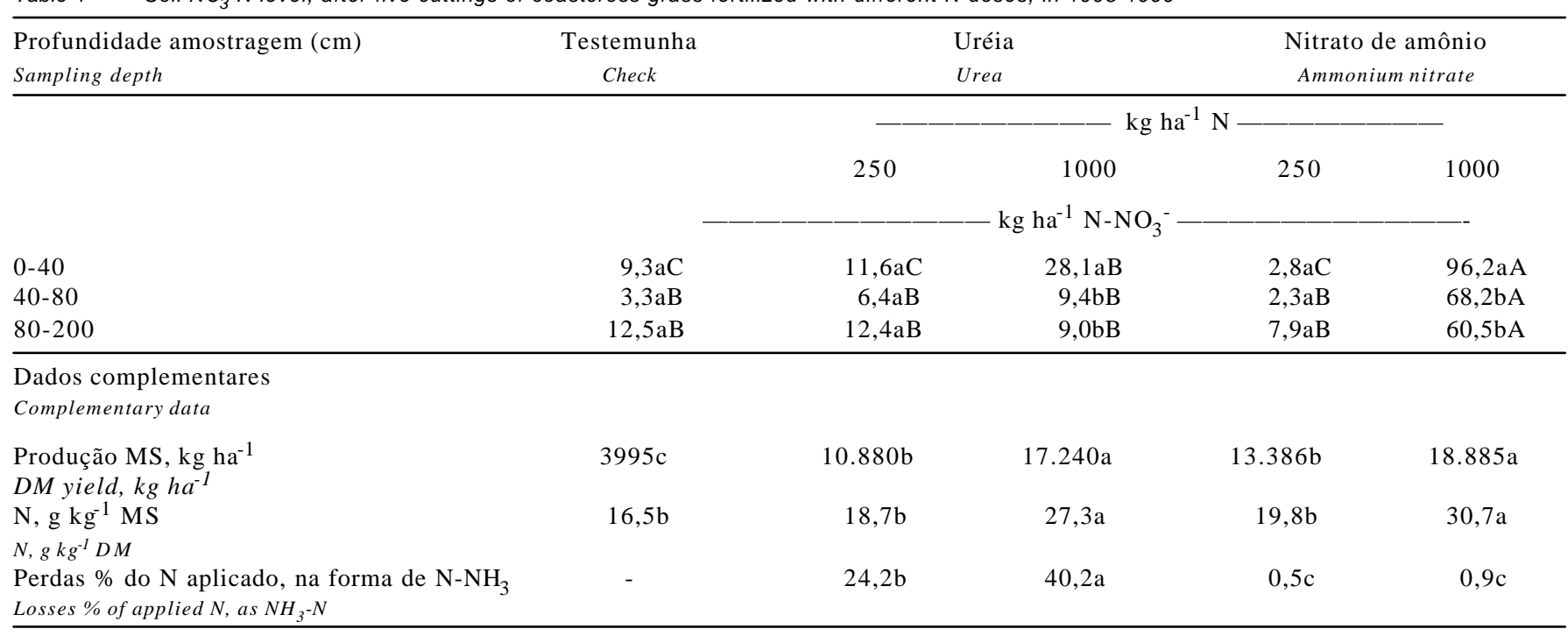

Médias seguidas de letras minúsculas (na coluna) e maiúsculas (na linha) diferentes diferem entre si $(P<0,05)$ pelos testes Tukey e Lsmeans, respectivamente. Means followed by different little (in column) and capital (in line) letters are different ( $P<0.05$, respectively, Tukey and Lsmeans) . 
et al., 2001b) em solos com bom suprimento de cálcio em profundidade ou sem limitações físicas.

Estudos com lisímetros em capins manejados sob corte têm mostrado que, quando nenhum fertilizante nitrogenado é aplicado, a lixiviação de N-nitrato é irrelevante (menor que 2,5 mg kg-1 ano $^{-1}$ ) (Low, 1973; Webster \& Dowdell, 1984). $\mathrm{O}$ mesmo ocorreu neste experimento, tanto no tratamento testemunha como nas parcelas adubadas, exceto com 1.000 $\mathrm{kg} \mathrm{ha}^{-1} \mathrm{ano}^{-1}$ de $\mathrm{N}$ na forma de nitrato de amônio, quando chegou a $2,8 \mathrm{mg} \mathrm{kg}^{-1}$ ou 5,6 $\mathrm{kg} \mathrm{ha}^{-1}$ versus $3 \mathrm{~kg} \mathrm{ha}^{-1} \mathrm{da}$ testemunha, abaixo de $140 \mathrm{~cm}$ de profundidade (Figura 1).

Di \& Cameron (2002) relataram perdas de $\mathrm{N}_{-} \mathrm{NO}_{3}{ }^{-}$no solo variando de 56 a $81 \mathrm{mg} \mathrm{kg}^{-1}$, dependendo da quantidade e das formas de $\mathrm{N}$ aplicado e das condições da pastagem, de modo que a aplicação de uréia $\left(200 \mathrm{~kg} \mathrm{ha}^{-1} \mathrm{ano}^{-1}\right.$ de N) contribuiu em pequena proporção no total das perdas de $\mathrm{N}-\mathrm{NO}_{3}{ }^{-}$por lixiviação.

A pequena lixiviação de $\mathrm{N}-\mathrm{NO}_{3}{ }^{-}$verificada no primeiro ano refere-se a amostragens de solo realizadas no final da época das águas, ressaltando-se que perdas maiores podem ter ocorrido durante o pico da estação das chuvas. Outra explicação para os teores relativamente baixos de $\mathrm{N}-\mathrm{NO}_{3}{ }^{-}$ no solo é o fato de as gramíneas tropicais serem excelentes extratoras de nitrogênio do solo (Primavesi et al., 2001a; Cantarella et al., 2002; Martha Jr. \& Vilela, 2002), em especial quando submetidas ao manejo rotacionado, com intensa atividade de morte-renovação do sistema radicular. O capim-coastcross, além de apresentar boa ocupação do

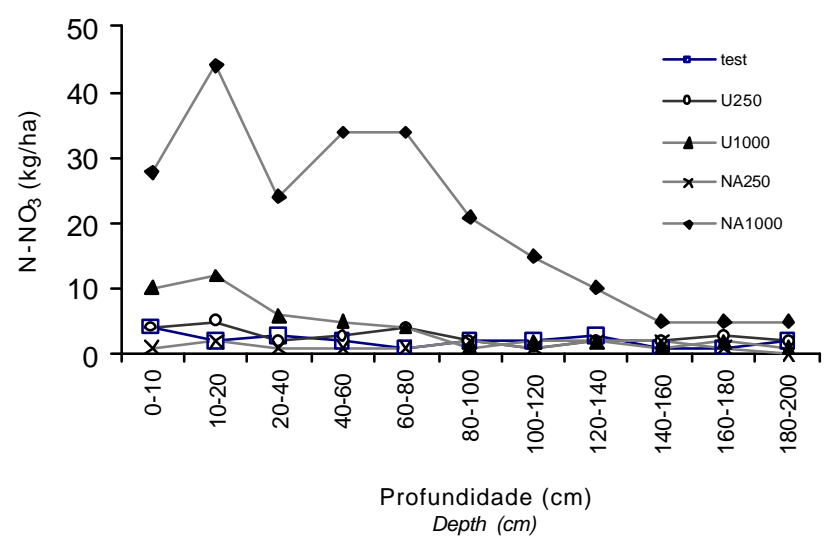

Figura 1 - Teores de $\mathrm{N}^{-} \mathrm{NO}_{3}{ }^{-}$no solo, de acordo com as doses de N, em 1998-1999. U250 e U1000 = 50 e $200 \mathrm{~kg} \mathrm{ha}^{-1}$ de $\mathrm{N}$-uréia por aplicação (cinco vezes). NA = $\mathrm{N}$-nitrato de amônio. Coeficiente de variação de $83 \%$ e diferença mínima significativa entre camadas de $4,3 \mathrm{~kg} \mathrm{ha}^{-1}$ e entre tratamentos de $2,6 \mathrm{~kg} \mathrm{ha}^{-1}$.

Figure 1 - Soil $\mathrm{NO}_{3}-\mathrm{N}$ levels, as function of $\mathrm{N}$ doses, in 1998-1990. U250 and $U 1000=50$ and $200 \mathrm{~kg} \mathrm{ha}^{-1}$ of urea- $\mathrm{N}$ per time (5 times). $N A=$ ammonium nitrate $-N$. Coeficient of Variation of $83 \%$ Least significant difference among soil layers of 4.3 and $2.6 \mathrm{~kg} \mathrm{ha}^{-1}$ among treatments. solo e grande consumo de N (Primavesi et al., 2004), também não libera $\mathrm{N}$ do material orgânico quando sua forragem cortada é imediatamente retirada do local monitorado. Ou tros autores também têm mostrado que aplicações inferiores a $10 \mathrm{mg} \mathrm{kg}^{-1} \mathrm{ano}^{-1}$ de $\mathrm{N}^{-N_{3}}{ }^{-}$são lixiviadas com doses menores que $250 \mathrm{~kg} \mathrm{ha}^{-1} \mathrm{ano}^{-1}$ de $\mathrm{N}$ (Barraclough et al., 1983;1984).

Os resultados obtidos neste experimento ratificam as afirmações de que quando as pastagens são bem manejadas, mesmo que intensivamente, parecem apresentar impacto ambiental positivo (Boddey et al., 1996), não se tornando preocupantes as perdas de $\mathrm{N}$ por lixiviação (Catchpoole, 1975; Catchpoole et al., 1983; Prasertsak et al., 2001) quando se aplicam quantidades de $\mathrm{N}$ normalmente absorvidas pelas plantas e quando o lençol freático não é superficial. Bartholomew (1971), no entanto, afirma que também pode ocorrer absorção do $\mathrm{N}$ do fertilizante pelas plantas forrageiras em profundidades de até $1,60 \mathrm{~m}$.

No segundo experimento, iniciado em novembro de 1999, as determinações de $\mathrm{N}-\mathrm{NO}_{3}{ }^{-}$, ,ealizadas em intervalos de sete dias (Figuras 2 a 4 ) durante todo o período das chuvas, excluindo-se as amostragens de 2/2/00 e 14/3/00 (dados perdidos), indicaram que os teores de $\mathrm{N}-\mathrm{NO}_{3}{ }^{-}$variaram $(\mathrm{P}<0,01)$ com as fontes e as doses de $\mathrm{N}$. Com o nitrato de amônio, os teores de $\mathrm{N}_{-} \mathrm{NO}_{3}{ }^{-}$se mostraram mais elevados em profundidade, especialmente na dose de $1.000 \mathrm{~kg} \mathrm{ha}^{-1}$ $\mathrm{ano}^{-1}$ de $\mathrm{N}$, fato também verificado por Cantarella et al. (2003), em uma cultura de citros.

A maior variação no teor de $\mathrm{N}-\mathrm{NO}_{3}{ }^{-}$ocorreu nas camadas superiores do solo ( $\mathrm{P}<0,01)$, até $20 \mathrm{~cm}$ de profundidade (Tabela 2), com tendência de movimentação do $\mathrm{N}-\mathrm{NO}_{3}{ }^{-}$para profundidades abaixo de $100 \mathrm{~cm}$, para as doses de $\mathrm{N}$ maiores que $500 \mathrm{~kg} \mathrm{ha}^{-1} \mathrm{ano}^{-1}$. Portanto, pode-se esperar baixo risco potencial de contaminação do lençol freático com $\mathrm{N}-\mathrm{NO}_{3}{ }^{-}$ em solos profundos com doses iguais ou inferiores a $500 \mathrm{~kg}$ $\mathrm{ha}^{-1} \mathrm{ano}^{-1}$ de N em pastagens. Segundo Corsi et al. (2001), a lixiviação de $\mathrm{N}^{-} \mathrm{NO}_{3}{ }^{-}$pode causar problemas em locais onde os índices pluviométricos são elevados e os solos são rasos, arenosos, com baixa capacidade de troca catiônica e mal manejados.

A partir da curva de variação de $\mathrm{N}_{-} \mathrm{NO}_{3}{ }^{-}$no tratamento testemunha, na camada 0-40 cm (Figura 2), que havia recebido em torno de $250 \mathrm{~kg} \mathrm{ha}^{-1}$ de $\mathrm{N}$ em cada um dos dois anos anteriores à instalação desse experimento, verificou-se crescimento gradual do conteúdo de $\mathrm{N}_{-} \mathrm{NO}_{3}{ }^{-}$até aproximadamente $100 \mathrm{~kg} \mathrm{ha}^{-1} \mathrm{com}$ o avançar do período das chuvas, com queda gradual no final desse período. Esse fato poderia ser resultante da mineralização de biomassa microbiana ou radicular; ou seja, resultado de atividade 
Tabela 2 - Média (22 semanas de amostragem) do teor de ${\mathrm{N}-\mathrm{NO}_{3}}^{-}$nos tratamentos e nas camadas de solo, 1999-2000 Table 2 - Mean (22 sampling weeks) of NO3-N level at different treatments and soil layer, 1999-2000

\begin{tabular}{|c|c|c|c|c|c|c|c|c|c|c|}
\hline \multirow[t]{2}{*}{$\begin{array}{l}\text { Tratamento } \\
\text { Treatment }\end{array}$} & \multirow{2}{*}{$\begin{array}{l}\text { Dose } \mathrm{N} \\
N \text { dose } \\
\mathrm{kg} \mathrm{ha}^{-1} \mathrm{ano}^{-1} \\
\text { kg hal }^{-1} \text { year }^{-1}\end{array}$} & \multicolumn{9}{|c|}{$\begin{array}{c}\text { Profundidade, } \mathrm{cm} \\
\text { Depth, } \mathrm{cm}\end{array}$} \\
\hline & & 10 & 20 & 40 & 60 & 80 & 100 & 120 & 140 & 160 \\
\hline & & & & & & & & & & \\
\hline $\begin{array}{l}\text { NA(AN) } \\
\text { Uréia (Urea) }\end{array}$ & $\begin{array}{l}1000 \\
1000\end{array}$ & $13 \mathrm{~b}$ & $\begin{array}{l}14 \mathrm{ab} \\
10 \mathrm{ab}\end{array}$ & $\begin{array}{r}10 \\
8\end{array}$ & $\begin{array}{l}8 \\
6\end{array}$ & $\begin{array}{l}8 \\
6\end{array}$ & $\begin{array}{l}0 \\
6\end{array}$ & $\begin{array}{l}6 \\
8\end{array}$ & $\begin{array}{l}6 \\
7\end{array}$ & 6 \\
\hline $\mathrm{NA}(A N)$ & 500 & $12 b$ & $9 b$ & 8 & 7 & 6 & 6 & 6 & 6 & 6 \\
\hline Uréia (Urea) & 500 & $10 \mathrm{~b}$ & $8 \mathrm{~b}$ & 7 & 8 & 8 & 7 & 6 & 7 & 7 \\
\hline Testemunha (Check) & 0 & $9 b$ & $7 \mathrm{~b}$ & 7 & 5 & 5 & 6 & 6 & 5 & 6 \\
\hline
\end{tabular}

$\mathrm{NA}=$ nitrato de amônio. Médias acompanhadas de mesmas letras não diferem entre si $(\mathrm{P}>0,01)($ Tukey), assim como as médias nas camadas inferiores a $20 \mathrm{~cm}$.

$A N=$ ammonium nitrate. Means followed by the same letter are not different ( $P>0.01)$ (Tukey), as well as the means bellow $20 \mathrm{~cm}$

biológica (fonte de N) no solo no período mais favorável após um período inicial consumidor e sua redução ou parada de atividade no período mais seco. Suhet et al. (1986) já haviam verificado que pode ocorrer concentração em torno de $42 \mathrm{~kg} \mathrm{ha}^{-1}$ de $\mathrm{N}-\mathrm{NO}_{3}{ }^{-} 30$ dias após o início das chuvas em Latossolo Vermelho Escuro virgem de cerrado. $\mathrm{O}$ crescimento em platôs nos tratamentos com adubo nitrogenado somente ocorreu nas camadas de 40 a $80 \mathrm{~cm}$ e de 80 a $160 \mathrm{~cm}$ (Figuras 3 e 4), de modo que, na camada de 0 a $40 \mathrm{~cm}$ (Figura 2), ocorreu sucessão quase regular de picos e reduções de nitrato ao longo do período das chuvas, sugerindo a possibilidade de ser conseqüência de acúmulo gradual de resíduo de $\mathrm{N}$ não absorvido pelas plantas forrageiras ou pela biomassa microbiana. Comparando aos dados de produção de matéria seca relatados por Primavesi et al. (2001a), surge a questão do não aumento da produção de forragem com aumento da oferta de nitrato no solo no final do período das chuvas, embora a maior produção de forragem, inclusive da testemunha ( $988 \mathrm{~kg} \mathrm{ha}^{-1}$ de MS), tenha ocorrido no terceiro período (11/1 a 14/2/2000), seguido de redução no período seguinte (testemunha com $332 \mathrm{~kg} \mathrm{ha}^{-1}$ de MS) e recuperação da produção no quinto e último períodos de corte (testemunha com $776 \mathrm{~kg} \mathrm{ha}^{-1}$ de MS). O quarto período em que ocorreu elevação do platô de N-NO3 coincidiu com o período de menor produção de forragem e o quinto período com produção maior correspondeu ao final do período de chuvas. Essa interação chuvas x umidade do solo $x$ atividade microbiológica e teor de $\mathrm{N}-\mathrm{NO}_{3}$ já havia sido observada por Suhet et al. (1986).

Torna-se, portanto, difícil identificar o fator que determina os picos de nitrato no solo, haja vista oen volvimento de fatores abióticos, como chuvas, períodos secos seguidos de chuva, variação de temperatura e aplicação de adubos nitrogenados, e de fatores bióticos, como decomposição de tecido vegetal ou fase fenológica, com desenvolvimento

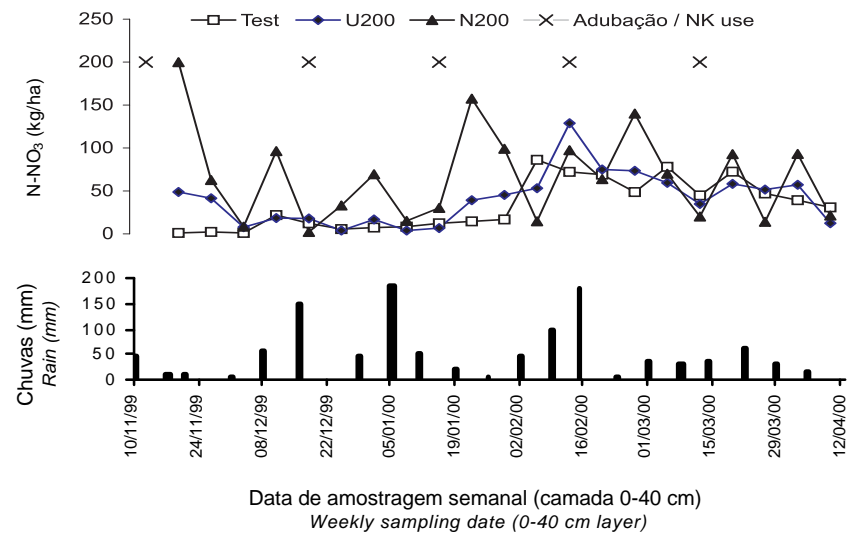

Figura 2 - Variação semanal no conteúdo de $N-\mathrm{NO}_{3}{ }^{-}$nacamada0-40 $\mathrm{cm}$ do solo. U200 = $200 \mathrm{~kg} \mathrm{ha}^{-1}$ de N-uréia por aplicação, NA200 = $200 \mathrm{~kg} \mathrm{ha}^{-1}$ de N-nitrato de amônio por aplicação. Test = testemunha sem $\mathrm{N}$ no período estudado, $\mathrm{x}$ = adubação com $\mathrm{NK}$ logo após corte da forrageira.

Figure 2 - Weekly variation of $\mathrm{NO}_{3}-\mathrm{N}$ content at the $0-40 \mathrm{~cm}$ soil layer.U200 = $200 \mathrm{~kg} \mathrm{ha}^{-1}$ pertime of urea-N, AN200 $=200 \mathrm{~kg} \mathrm{ha}^{-1}$ pertime of ammonium nitrate- $N$, Test $=$ plot without $N, x=$ use of $N K$ fertilizer, soon after forage cutting.
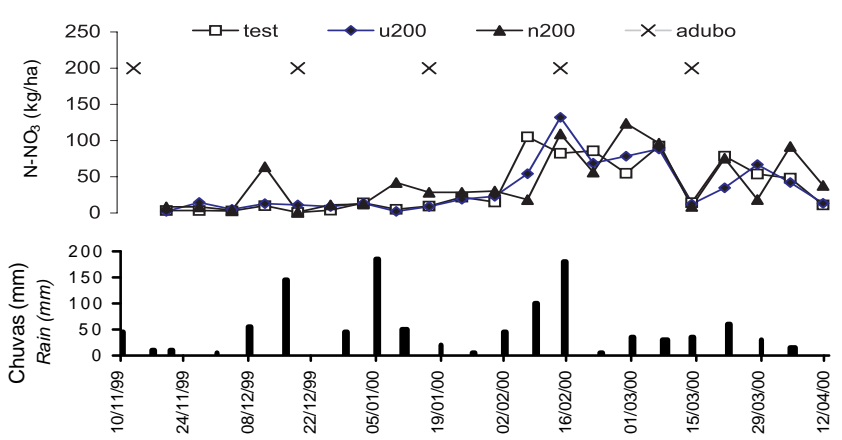

Data de amostragem semanal (camada $40-80 \mathrm{~cm}$ ) Weekly sampling date $(40-80 \mathrm{~cm}$ layer $)$

Figura 3 - Variação semanal no conteúdo de $\mathrm{N}^{-\mathrm{NO}_{3}}{ }^{-}$na camada 40 a $80 \mathrm{~cm}$ do solo, com $200 \mathrm{~kg} \mathrm{ha}^{-1}$ aplicação- ${ }^{-1}$ de N-uréia (U200) e N-nitrato de amônio (N200) ao longo do período chuvoso. Test $=$ testemunhasem $N$ no período estudado, $\mathrm{x}=$ adubação com NK, logo após corte da forrageira.

Figure 3 - Weekly variation of $\mathrm{NO}_{3}-\mathrm{N}$ content at the $40-80 \mathrm{~cm}$ soil layer, with $200 \mathrm{~kg} \mathrm{ha}^{-1}$ cutting $^{-1}$ of urea-N (U200) and ammonium nitrate-N (N200), along the rain season. Test $=$ plot without $N, x=$ use of NKfertilizer, soon after forage cutting. 
mais intenso da forrageira e biomassa microbiana do solo, constituindo os sumidouros ou "sinks" de nitrogênio, e morte de biomassa microbiana e mineralização de matéria orgânica, constituindo as fontes de N. Kissel \& Smith (1978) tentaram definir mais claramente os fatores responsáveis pela baixa recuperação do $\mathrm{N}$ e pelo desaparecimento do $\mathrm{N}-\mathrm{NO}_{3}{ }^{-}$em solos com argila expandida cobertos com capimcoastal bermuda e relataram que a imobilização foi o principal fator da baixa recuperação de $\mathrm{N}$, de modo que a maior parte do $\mathrm{N}$ residual no final da primeira estação de crescimento se apresentava como $\mathrm{N}$ imobilizado ou estava presente no sistema radicular do capim, o que, neste estudo, poderia ser representado pelas 12 semanas iniciais para a testemunha e as nove primeiras para os tratamentos com $\mathrm{N}$, em especial na camada superficial (Figura 2).

Na Figura 2 são ilustrados diferentes sinais de interferência na dinâmica do $\mathrm{N}-\mathrm{NO}_{3}{ }^{-}$no perfil do solo. Os picos na camada superficial $(0 \mathrm{a} 40 \mathrm{~cm}$ ) parecem ocorrer preferencialmente após eventos pluviais, sobretudo aqueles precedidos de períodos secos, dentro do período chuvoso, in de pendentemente da aplicação de adubos nitrogenados, su gerindo que, nos períodos mais úmidos, ocorre grande absorção de $\mathrm{N}-\mathrm{NO}_{3}{ }^{-}$pela gramínea forrageira no período de desenvolvimento mais intenso, quando podem desenvolver coroa e raízes em profundidade, além de imobilização pela população microbiana do solo.

Essa última suspeita é reforçada pelo mesmo padrão instantâneo do perfil de distribuição de nitrato em todo o perfil ao longo das semanas (Figuras 3 e 4), embora esse fenômeno possa resultar de movimentação rápida de nitrato solúvel ao longo do perfil, dependendo do volume de chuvas no período. Além disso, as parcelas que receberam $\mathrm{N}$-uréia não apresentaram redução no teor de $\mathrm{N}_{-} \mathrm{NO}_{3}{ }^{-}$, apesar da média de perda de $28 \%$ de $\mathrm{N}$ por volatilização de amônia, como relatado por Primavesi et al. (2001a), reforçando a idéia de participação complementar do $\mathrm{N}$ microbiano e da mineralização de matéria orgânica do solo.

Neste segundo experimento, o maior teor de $\mathrm{N}_{-} \mathrm{NO}_{3}{ }^{-}$no solo foi de $81,2 \mathrm{mg} \mathrm{kg}^{-1}$, no início das águas, na camada de 0 a $10 \mathrm{~cm}$, na parcela que recebeu $1.000 \mathrm{~kg} \mathrm{ha}^{-1} \mathrm{de} \mathrm{N}$ na forma de nitrato de amônio. Os baixos teores de $\mathrm{N}-\mathrm{NO}_{3}{ }^{-}$no perfil do solo confirmam o resultado obtido no experimento do ano anterior, podendo ser parcialmente explicados pela elevada capacidade de extração de N pelas gramíneas tropicais, em virtude de seu elevado potencial de produção de matéria seca.

Mesmo com a aplicação de doses elevadas de nitrogênio em pastagens de capim-coastcross, não houve perda intensa de nitrato em profundidade, à semelhança do ocorrido na
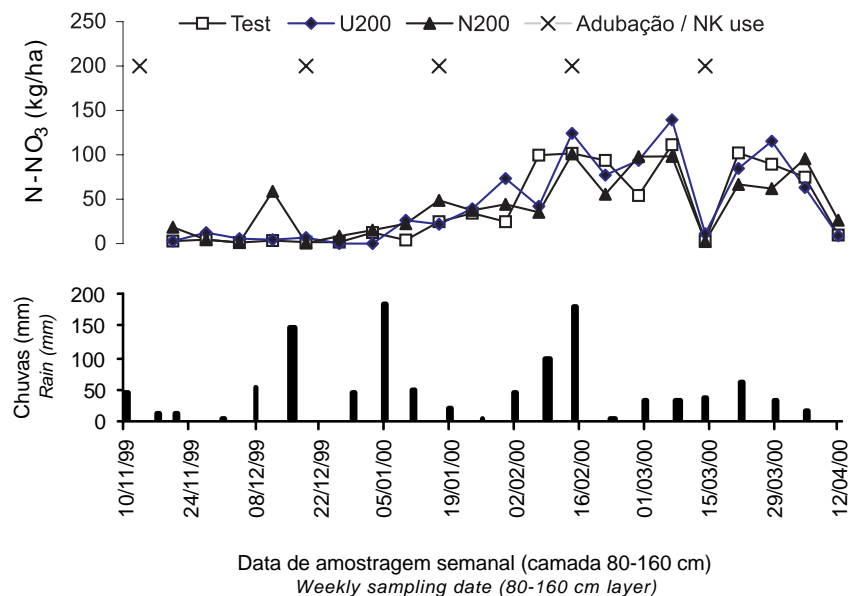

Figura 4 - Variação semanal no conteúdo de $\mathrm{N}^{-\mathrm{NO}_{3}}{ }^{-}$na camada 80 a $160 \mathrm{~cm}$ do solo, com $200 \mathrm{~kg} \mathrm{ha}^{-1}$ aplicação-1 de $\mathrm{N}$-uréia (U200) e N-nitrato de amônio (N200), ao longo do período chuvoso. Test = testemunha sem $\mathrm{N}$ no período estudado, $x$ = adubação com NK logo após corte da forrageira.

Figure 4 - Weekly variation of $\mathrm{NO}_{3}-\mathrm{N}$ content at the $80-160 \mathrm{~cm}$ soil layer, with $200 \mathrm{~kg} \mathrm{ha}^{-1}$ of urea-N (U200) and ammonium nitrate- $N$ (N200), along the rain season. Test $=$ plot without $N, x=$ use of NK fertilizer, soon after forage cutting.
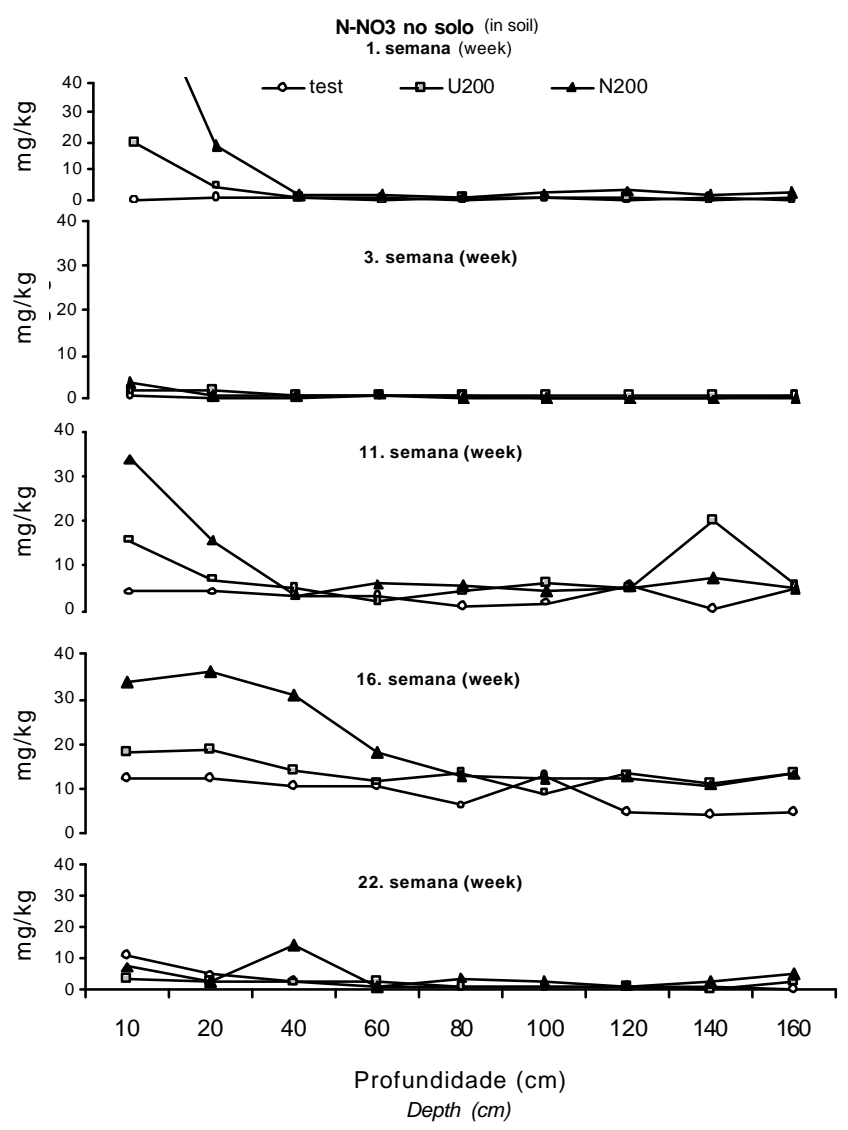

Figura 5 - Variação dos teores de $\mathrm{N}-\mathrm{NO}_{3}{ }^{-}$nas camadas de solo com $200 \mathrm{~kg} \mathrm{ha}^{-1}$ aplicação ${ }^{-1}$ de $\mathrm{N}$-uréia (U200) e $\mathrm{N}$-nitrato de amônio (N200) ao longo do período chuvoso, em semanas consecutivas.

Figure 5 - $\quad \mathrm{NO}_{3}-\mathrm{N}$ variation, in soil layer, with $200 \mathrm{~kg} \mathrm{ha}^{-1}$, after each cutting, of urea-N (U200) and ammonium nitrate- $N$ (N200), along the rain season, in consecutive weeks. 
área testemunha (Tabela 2). Em $160 \mathrm{~cm}$ de profundidade, a média da diferença entre a testemunha e as adubadas não passou de $1 \mathrm{mg} \mathrm{kg}^{-1}$ de solo, embora possam ocorrer picos no teor de nitrato (Figura 5) ao longo do período das chuvas, indicando não haver perigo iminente para a qualidade ambiental em solos profundos, sem afloramento de lençol freático, como próximo de nascentes, especialmente quando se considera que as doses normalmente utilizadas ficam entre 250 e $500 \mathrm{~kg} \mathrm{ha}^{-1}$ de N, parceladas em cinco vezes após cada corte ou pastejo consecutivo no período das chuvas. São necessários estudos por períodos mais longos para se verificar a possibilidade de aumento no excedente de nitrato no solo, o que sinalizaria a necessidade de redução das doses de adubo nitrogenado em anos consecutivos.

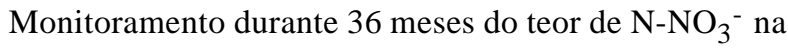
água, em diferentes corpos de água na bacia hidrográfica do Ribeirão Canchim (Primavesi et al., 2002), onde foram realizados esses experimentos, indicou haver variação entre 0,03 e $5,1 \mathrm{mg} \mathrm{L}^{-1}$, com valores médios entre 0,17 e $0,73 \mathrm{mg} \mathrm{L}^{-1}$, observando-se que a resolução do CONAMA número 20 (Agudo, 1987) tolera até $10 \mathrm{mg} \mathrm{L}^{-1}$. Constatou-se que parte desse nitrato originou-se da decomposição de matéria orgânica da serapilheira, sendo medido pico de $0,84 \mathrm{mg} \mathrm{L}^{-1}$ de nitrato em nascente dentro de mata mesófila semidecídua.

\section{Conclusões}

Doses de até $500 \mathrm{~kg} \mathrm{ha}^{-1}$ de nitrogênio, parceladas em cinco vezes, na forma de uréia ou de nitrato de amônio, no período das chuvas, em pastagem de capim-coastcross, não proporcionam perdas significativas de nitrato para o lençol freático.

As grandes variações de nitrato nas doses de fertilizantes nitrogenados normalmente praticadas em pastagens ocorrem na camada de 0 a $40 \mathrm{~cm}$ e se estabilizam em torno de $100 \mathrm{~cm}$ profundidade.

\section{Literatura Citada}

AGUDO, E.G. Guia de coleta e preservação de amostras de água. 1.ed. São Paulo: Companhia de Tecnologia de Saneamento Ambiental - CETESB, da Secretaria do Meio Ambiente do Governo de São Paulo, 1987. 150p.

BARTHOLOMEW, W.V. ${ }^{15} \mathrm{~N}$ in research on the availability and crop use of nitrogen. In: INTERNATIONAL ATOM ENERGY AGENCY, NITROGEN-15 IN SOIL-PLANT STUDIES, 1971, Viena. Proceedings... Viena: IAEA, 1971. p.1-20. (PL-341/1)

BARRACLOUGH, D.; HYDEN, M.J.; DAVIES, G.P. Fate of fertilizer nitrogen applied to grassland. I. Field leaching results.Journal of Soil Science, v.34, p.483-497, 1983.

BARRACLOUGH, D.; GEENS, E.L.; MAGGS, J.M. Fate of fertilizer nitrogen applied to grassland. II. Nitrogen-15 leaching results. Journal of Soil Science, v.35, p.191-199, 1984.
BODDEY, R.M.; RAO, I.M.; THOMAS, R.J. Nutrient cycling and environmental impact of Brachiaria pastures. In: WILES, J.W.; MAASS, B.L.; VALLE, C.B. (Eds.) Brachiaria: biology, agronomy and improvement. Cali: CIAT, 1996. p.72-86. (CIAT Publications, 259).

CAMERON, K.C.; DI, H.J.; MCLAREN, R.G. Is soil an appropriate dumping ground for our wastes? Australian Journal of Soil Research, v.35, p.995-1035, 1997.

CANTARELLA, H.; MATTOS JR., D.; QUAGGIO, J.A. et al. Fruit yield of Valencia sweet orange fertilized with different $\mathrm{N}$ sources and the loss of applied $\mathrm{N}$. Nutrient Cycling in Agroecosystems, v.67, p.215-223, 2003

CANTARELLA, H.; CORRÊA, L.A.; PRIMAVESI, O. et al. Fertilidade do solo em sistemas intensivos de manejo de pastagens. In: SIMPÓSIO SOBRE MANEJO DA PASTAGEM, 19., 2002, Piracicaba. Anais... Piracicaba: Fundação de Estudos Agrários Luiz de Queiroz, 2002. p.99-132.

CATCHPOOLE, V.R. Pathways for losses of fertilizer nitrogen from a Rhodes grass pasture in south-eastern Queensland. Australian Journal of Agricultural Research, v.26, p.259-268, 1975.

CATCHPOOLE, V.R.; OXENHAM, D.J.; HARPER, L.A. Transformation and recovery of urea applied to a grass pasture in south-eastern Queensland. Australian Journal of Experimental Agricultural and Animal Husbandry, v.23, p.80-86, 1983.

CORSI, M. Pastagem de alta produtividade. In: SIMPÓSIO SOBRE MANEJO DE PASTAGENS, 8., 1986, Piracicaba. Anais... Piracicaba: Fundação de Estudos Agrários Luiz de Queiroz, 1986, p.499-512.

CORSI, M.; MARTHA JR., G.B.; BASALOBRE, M.A.A. et al. Tendências e perspectivas da produção de bovinos sob pastejo. In: SIMPÓSIO SOBRE MANEJO DA PASTAGEM, 17., 2001. Piracicaba. Anais... Piracicaba: Fundação de Estudos Agrários Luiz de Queiroz, 2001. p.3-69.

DI, H.J.; CAMERON, K.C. Nitrate leaching and pasture production from different nitrogen sources on a shallow stoney soil under flood-irrigated dairy pastue. Australian Journal of Soil Research, v.40, p.317-334, 2002.

ERREBHI, M.; ROSEN, C.J.; GUPTA, S.C. et al. Potato yield response and nitrate leaching as influenced by nitrogen management. Agronomy Journal, v.90, n.1, p.10-15, 1998.

FAVORETTO, V.; CECATO, U.; GUIDELI, C. et al. Avaliação da estrutura de vegetação do capim-colonião em função de práticas de manejo. In: REUNIÃO ANUAL DA SOCIEDADE BRASILEIRA DE ZOOTECNIA, 32., 1995, Brasília. Anais... Brasília: Sociedade Brasileira de Zootecnia, 1995. p.45-48.

GATES, C.E. A user's guide to misanalyzing planned experiments. HortScience, v.26, n.10, p.1262-1265, 1991.

GOMES, J.F.; SIEWERDT, L.; SILVEIRA JR., P. Avaliação da produtividade e economicidade do feno de capim-pangola (Digitaria decumbens Stent) fertilizado com nitrogênio. Revista da Sociedade Brasileira de Zootecnia, v.16, n.6, p.491-499, 1987.

GRANLI, T.; BOCKMAN, O.C. Nitrous oxide from agriculture. Norwegian Journal of Agricultural Science, v.12, p.1128, 1994 (suppl. 2).

KISSEL, D.E.; SMITH, S.J. Fate of fertilizer nitrate applied to coastal bermudagrass on a swelling clay soil. Soil Science Society American Journal, v.42, p.77-80, 1978.

LOW, A.J. Nitrate and ammoniun nitrogen concentration in water draining through soil monolits in lysimeters cropped with grass or clover or uncropped. Journal of Agricultural Science of Food and Agriculture, v. 24, p.1489-1495, 1973.

MARTHA JR., G.B.; VILELA, L. Pastagens no Cerrado: baixa produtividade pelo uso limitado de fertilizantes em pastagens. Planaltina: Embrapa Cerrados, 2002. 32p. (Documentos, 50).

MELLO, F.A.F.; BRASIL SOBRINHO, M.O.C.; ARZOLLA, S. et al. Fertilidade do solo. 2.ed. São Paulo: Nobel, 1984. 400p. 
MELLO, F.A.F. Uréia fertilizante. Campinas: Fundação Cargill, 1987. 192p.

PRASERSAK, P.; FRENEY, J.R.; DENMEAD, O.T. et al. Significance of gaseous nitrogen loss from a tropical dairy pasture fertilized with urea. Australian Journal of Experimental Agriculture, v.41, p.625-632, 2001.

PRIMAVESI, O.; CORRÊA, L.A.; PRIMAVESI, A.C. et al. Adubação com uréia em pastagem deCynodon dactyloncv. Coastcross: eficiência e perdas. São Carlos: Embrapa Pecuária Sudeste, 2001a. 42p. (Circular Técnica, 30)

PRIMAVESI, O.; FREITAS, A.R.; PRIMAVESI, A.C.P.A. et al Qualidade ambiental em sistema intensivo de produção de bovinos de leite, na microbacia do ribeirão Canchim: indicadores, manejo e problemas. São Carlos: Embrapa Pecuária Sudeste, 2001b. 70p. (Boletim de Pesquisa, 07)

PRIMAVESI, O.; FREITAS, A.R.; PRIMAVESI, A.C. et al. Water quality of the Canchim's creek watershed, in São Carlos, SP, Brazil, occupied by beef and dairy cattle activities. Brazilian Archives of Biology and Technology, v.45, n.2, p.209-217, 2002.

PRIMAVESI, A.C.; PRIMAVESI, O.; CORRÊA, L.A. et al. Adubação nitrogenada em capim-coastcross: efeitos na extração de nutrientes e recuperação aparente do nitrogênio. Revista Brasileira de Zootecnia, v.33, n.1, p.68-78, 2004.

SUHET, A.R.; PERES, J.R.R.; VARGAS, M.A.T. Nitrogênio. In: GOEDERT, W.J. (Ed.) Solos dos cerrados: tecnologias e estratégias de manejo. São Paulo: Nobel; Brasília: Embrapa, Centro de Pesquisa Agropecuária dos Cerrados, 1985. p.167-202.

TEDESCO, M.J.; VOLKWEISS, S.J.; BOHNEN, H Análises de solo, plantas e outros minerais. Porto Alegre: Universidade Federal do Rio Grande do Sul, 1985. 188p. (Boletim Técnico de Solos, 5)

VICENTE-CHANDLER, J.; SILVA, S.; FIGARELLA, J. The effect of nitrogen fertilization and frequency of cutting on the yield and composition of three tropical grasses. Agronomy Journal, v.51, n.4, p.202-206, 1959

WEBSTER, C.P.; DOWDELL, R.J. Effect of drought and irrigation on the fate of nitrogen applied to cut permanent grass swards in lysimeters: leaching losses. Journal Science Food Agricultural, v.35, p.1105-1111, 1984.

WHITEHEAD, D.C. Volatilization of ammonia. In: WHITEHEAD, D.C. (Ed.) Grassland nitrogen. Wallingford: CAB International, 1995. p.152-179. 